\title{
Secuencia de bandas amnióticas, una actualización
} An update on amniotic bands sequence

\author{
Dra. Eunice López-Muñoz y Dr. Luis E. Becerra-Solano ${ }^{a}$
}

\section{RESUMEN}

La secuencia de bandas amnióticas es un desorden congénito caracterizado por anomalías craneofaciales, de la pared corporal y de las extremidades que pueden asociarse con bandas fibrosas fetoplacentarias. Su prevalencia ha sido reportada entre 0,19 y 8,1 por 10000 nacimientos. Diversas teorías han tratado de explicar su etiología, sin embargo, ninguna, en forma individual, sustenta todas y cada una de las anomalías observadas, por lo que se ha considerado una entidad multifactorial. La identificacióndeanomalías (pre-yposnatalmente) sugestivas de secuencia de bandas amnióticas permite el abordaje diagnóstico para efectuar intervenciones terapéuticas oportunas que posibiliten la liberación de bandas amnióticas mediante fetoscopia con recuperación de la perfusión de la porción distal de la extremidad involucrada o bien la reparación quirúrgica posnatal y para otorgar asesoramiento genético. Este artículo ofrece una actualización sobre aspectos epidemiológicos, teorías etiológicas, factores de riesgo, características clínicas, diagnóstico (queincluyeel diagnóstico prenatal), asesoramiento genético, abordaje terapéutico y pronóstico de esta entidad.

Palabras clave: bandas amnióticas, anillo de constricción.

http: / / dx.doi.org/10.5546/ aap.2018.e409

Texto completo en inglés:

a. Unidad de Investigación Médica en Medicina Reproductiva, Unidad Médica de Alta Especialidad. Hospital de Ginecoobstetricia $\mathrm{N}^{\circ} 4$ Luis Castelazo Ayala, Instituto Mexicano del Seguro Social, Ciudad de México.

\section{Correspondencia:} Dra. Eunice López-Muñoz: astridkaryme2001@ yahoo.com.mx

Financiamiento: Ninguno.

Conflicto de intereses: Ninguno que declarar.

Recibido: 6-7-2017 Aceptado: 3-10-2017 Arch Argent Pediatr 2018;116(3):e409-e420.

\section{INTRODUCCIÓN}

La secuencia de bandas amnióticas (Amniotic Band Sequence; ABS, por sus siglas en inglés) (OMIM $\% 217100)^{1-3}$ es un desorden congénito con anomalías fetales asociadas a bandas fibrosas fetoplacentarias deformación o malformación ${ }^{4} \sin$ un patrón anatómico consistente. ${ }^{5,6}$ Las anomalías se limitan a estructuras externas con o sin disrupción de la pared corporal o malformaciones internas que varían en gravedad y localización..$^{7-9}$ Algunos autores consideran por separado la ABS del complejo pared corporalextremidades (Limb Body Wall Complex; LBWC, por sus siglas en inglés), defectos de la pared corporal (Body Wall Defects; BWD, por sus siglas en inglés) o complejo pared corporal (Body Wall Complex; BWC, por sus siglas en inglés), ${ }^{10-12}$ mientras que otros los consideran parte de la misma enfermedad. ${ }^{13-16}$

\section{Clasificación}

Aun cuando se han propuesto diversas clasificaciones (Tabla 1), la European Surveillance of Congenital Anomalies (EUROCAT) sugiere mencionar el término "bandas de constricción congénitas" o "bandas amnióticas" y acompañarlo de la descripción específica de cada anomalía congénita identificada. ${ }^{22,23}$

\section{Epidemiología}

La prevalencia de ABS con o sin Cómo citar: López-Muñoz E, Becerra-Solano LE. el LBWC o BWD es variable, pues Secuencia de bandas amnióticas, una actualización. $\quad$ se reporta de 0,1924 a 8,125 por que pueden conducir a disrupción, 10000 nacimientos. Esta variabilidad radica en diferentes definiciones, terminología, clasificación y población de estudio (Tabla 2).

La prevalencia es mayor en fetos de 9-20 semanas de gestación $(178,5 \text { en } 10000 \text { casos })^{2}$ y en óbitos (191 en 10000 óbitos). ${ }^{31}$ Asumiendo que, aproximadamente, $20 \%$ de los embarazos identificados se pierden antes de las 20 semanas de gestación y $1 \%$ después de las 20 , un estudio estimó que, de cada 100 casos con ABS, alrededor de 90 se abortaban espontáneamente, 5 eran óbitos y 5 nacían vivos, por lo que su frecuencia había sido subestimada. ${ }^{31}$ 
TABLA 1. Clasificaciones propuestas para la secuencia de bandas amnióticas

\begin{tabular}{lc} 
Referencia & Bases para la clasificación \\
\hline Pagon et al., $1979^{17}$ & $\begin{array}{l}\text { Localización y extensión del defecto de la } \\
\text { pared corporal y características asociadas }\end{array}$
\end{tabular}

Seeds et al., $1982^{5}$

Van Allen et al., $1987^{18}$

Moerman et al., $1992^{19}$

Russo et al., $1993^{20}$

Martínez-Frías, 1997,11

y Martínez-Frías, $2000^{12}$

Jamsheer et al., 2009 10

Guzmán et al., 2013²1

Lowry et al., $2017^{16}$
Región anatómica en la que se ubica el defecto

Tipo de anomalías en las extremidades

Presencia de bandas de constricción y defectos asociados

LBWC y anomalías asociadas

Presencia o no de BWD

Presencia o no de BWD

Localización de los defectos

Presencia de bandas amnióticas y localización de los defectos
Grupos

Toraco- y/o abdominosquisis (fisura congénita de la línea media de la pared torácica y / o abdominal con protrusión visceral) con malformación craneal mayor, hendiduras faciales y bandas amnióticas

Defectos de la línea media con la extremidad inferior derecha ausente/rudimentaria, aplasia/hipoplasia renal derecha y anomalías genitales

Defectos de la pared abdominal lateral con anomalías del sistema nervioso central, genitales externos, ano imperforado y cordón umbilical corto

En las extremidades

Craneofaciales

Viscerales, que incluyen onfalocele y gastrosquisis

Secundarias a la disrupción vascular y de tejido subyacente

Secundarias a las bandas amnióticas o adhesiones Secundarias a las deformaciones vs. hemorragias

Bandas de constricción

Bandas de constricción y defectos craneofaciales Defectos complejos, que incluyen defectos de la pared corporal con evisceración de órganos torácicos o abdominales (BWD o BWC)

LBWC con excencefalia/encefalocele, hendiduras faciales y bandas amnióticas o adhesión entre el defecto craneal y la placenta

LBWC sin defectos craneofaciales, pero con anomalías urogenitales, atresia anal, meningocele lumbosacro, cordón umbilical corto o persistencia de celoma extraembrionario

ABS con defecto de las extremidades, craneofacial o constricción digital

BWD de cualquier causa (excepto onfalocele y gastrosquisis), que incluyen bandas amnióticas

- Hendiduras graves de la pared abdominal, cordón umbilical corto/ausente o que se prolonga a la placenta

ABS con BWD

$\mathrm{ABS} \sin \mathrm{BWD}$

Defectos craneofaciales y de las extremidades Defectos craneofaciales, de las extremidades y BWD

Defectos de las extremidades y BWD

Defectos aislados craneofaciales, de las extremidades o BWD con la presencia de bandas amnióticas

Bandas amnióticas/anillos de constricción con o sin deficiencia de las extremidades BWD con o sin deficiencia de las extremidades, con o sin bandas amnióticas/anillos de constricción Bandas amnióticas / anillos de constricción con defectos craneofaciales

Bandas amnióticas/anillos de constricción con deficiencia de las extremidades y defectos craneofaciales

Bandas amnióticas / anillos de constricción, defectos craneofaciales y BWD

ABS: secuencia de bandas amnióticas; BWD: defectos de la pared corporal; BWC: complejo de la pared corporal; LBWC: complejo pared corporal-extremidades. 


\section{Etiología}

Teoría de la displasia embrionaria (endógena) y teorías derivadas

Streeter ${ }^{32}$ sugirió que las bandas amnióticas surgían tempranamente en el desarrollo y no eran la causa primaria de los defectos, sino una consecuencia de la histogénesis imperfecta, que causaba necrosis, cicatrices, constricciones y fusiones, y que la deficiencia de una o dos células resultaba en una anomalía mayor en la estructura final. ${ }^{32,33} \mathrm{McKenzie}^{34}$ apoyó esta teoría sugiriendo que el tejido alterado era una muestra de la distribución anormal de las áreas de muerte celular. ${ }^{33-36}$

Hartwig y cols., ${ }^{37}$ sugirieron que las anomalías en las extremidades y en la pared lateral corporal se debían a defectos en las placodas ectodérmicas, que resultaban en la deficiencia mesodérmica $y$, por lo tanto, de la pared abdominal, la cual era sustituida por el amnios, que, ante la ruptura,

TABLA 2. Prevalencia de la secuencia de bandas amnióticas en diferentes poblaciones

\begin{tabular}{|c|c|c|c|c|}
\hline Referencia & Fuente de información & Período de estudio & Tasa reportada & Otros hallazgos reportados \\
\hline $\begin{array}{l}\text { Garza et al., } \\
1988^{15}\end{array}$ & $\begin{array}{c}\text { Metropolitan Atlanta Congenital } \\
\text { Defects Program, } \\
\text { Estados Unidos de América }\end{array}$ & 1968-1982 & 1,16 por $10000 \mathrm{RNV}$ & $\begin{array}{c}\text { Relación 1:1,5 } \\
\text { (femenino/masculino). }\end{array}$ \\
\hline $\begin{array}{l}\text { Bower et al., } \\
1993^{26}\end{array}$ & $\begin{array}{c}\text { Wester Australia } \\
\text { Birth Defects Registry, Australia } \\
\text { South Australia } \\
\text { Birth Defects Registry, Australia }\end{array}$ & $\begin{array}{l}1986-1989 \\
1986-1990\end{array}$ & 2,03 por $10000 \mathrm{RNV}$ & $\begin{array}{c}\text { Ambos sexos } \\
\text { igualmente afectados. }\end{array}$ \\
\hline $\begin{array}{l}\text { Czeizel et al., } \\
1987^{27}\end{array}$ & $\begin{array}{l}\text { Hungarian Congenital } \\
\text { Abnormality Registry, Hungría }\end{array}$ & 1975-1984 & 1,31 por $10000 \mathrm{RNV}$ & $\begin{array}{l}\text { Ambos sexos igualmente } \\
\text { afectados. }\end{array}$ \\
\hline $\begin{array}{l}\text { Froster et al., } \\
1993^{24}\end{array}$ & $\begin{array}{c}\text { British Columbia Health } \\
\text { Surveillance Registry, Canadá }\end{array}$ & $\begin{array}{r}\text { 1952-1984 } \\
\text { prese }\end{array}$ & $\begin{array}{l}0,19 \text { por } 10000 \mathrm{RNV} \\
\text { acluyeron casos sin defecto } \\
\text { las extremidades aun en } \\
\text { cia de anillos de constricción) }\end{array}$ & $\begin{array}{l}3 \text { de los casos reportados } \\
\text { fueron considerados de } \\
\text { tipo familiar. }\end{array}$ \\
\hline $\begin{array}{l}\text { Martínez-Frías, } \\
1997^{11}\end{array}$ & $\begin{array}{c}\text { Estudio Colaborativo Español } \\
\text { de Malformaciones } \\
\text { Congénitas, España }\end{array}$ & 1976-1996 & 0,59 por $10000 \mathrm{RNV}$ & $\begin{array}{l}\text { Mayor frecuencia de bandas } \\
\text { amnióticas aisladas en el sexo } \\
\text { femenino y de otras anomalías } \\
\text { o BWD en el sexo masculino. }\end{array}$ \\
\hline $\begin{array}{l}\text { Luehr et al., } \\
2002^{28}\end{array}$ & $\begin{array}{l}\text { Centro de referencia de } \\
\text { tercer nivel, Australia }\end{array}$ & 1996-2001 & $\begin{array}{c}3,3 \text { por } 10000 \mathrm{RNV} \\
\text { y abortos }\end{array}$ & $\begin{array}{l}\text { Casos que cumplieran con los } \\
\text { criterios de LBWC. } \\
1 \text { de los casos reportados } \\
\text { fue considerado familiar. } \\
\text { La prevalencia no está } \\
\text { calculada sobre la base de } \\
\text { un registro poblacional. }\end{array}$ \\
\hline $\begin{array}{l}\text { Orioli et al., } \\
2003^{29}\end{array}$ & $\begin{array}{l}\text { Estudio Colaborativo Latino- } \\
\text { Americano de Malformaciones } \\
\text { Congénitas, Sudamérica }\end{array}$ & 1982-1998 & $\begin{array}{l}0,97 \text { por } 10000 \mathrm{RNV} \\
\text { y óbitos }\end{array}$ & $\begin{array}{l}8 \text { de los casos reportados } \\
\text { fueron considerados de } \\
\text { tipo familiar. }\end{array}$ \\
\hline $\begin{array}{l}\text { Jamsheer et al., } \\
2009^{10}\end{array}$ & $\begin{array}{l}\text { Polish Registry of } \\
\text { Congenital Malformations } \\
\text { (PRCM), Polonia }\end{array}$ & 1998-2006 & $\begin{array}{c}0,29 \text { por } 10000 \mathrm{RNV} \\
\text { y óbitos }\end{array}$ & $\begin{array}{l}\text { Mayor gravedad y frecuencia } \\
\text { de anomalías en los } \\
\text { pacientes con BWD. }\end{array}$ \\
\hline $\begin{array}{l}\text { Koskimies et al., } \\
2015^{30}\end{array}$ & $\begin{array}{l}\text { Registros poblacionales de } \\
\text { Finlandia, Finlandia }\end{array}$ & 1993-2005 & $\begin{array}{c}0,9 \text { por } 10000 \mathrm{RNV} \\
\text { y óbitos }\end{array}$ & $\begin{array}{c}\text { Relación 1:1,46 } \\
\text { (femenino/masculino). }\end{array}$ \\
\hline $\begin{array}{l}\text { Lowry et al., } \\
2017^{16}\end{array}$ & $\begin{array}{l}\text { Alberta Congenital Anomalies } \\
\text { Surveillance System, Canadá }\end{array}$ & 1980-2012 óbito & $\begin{array}{l}1,08 \text { por } 10000 \mathrm{RNV} \text {, } \\
\text { interrupciones del embarazo } \\
20 \text { semanas de gestación) }\end{array}$ & $\begin{array}{c}\text { Relación 1:1,15 } \\
\text { (femenino/masculino). } \\
\text { Mayor gravedad de los } \\
\text { defectos en las extremidades } \\
\text { en los pacientes con BWD. }\end{array}$ \\
\hline $\begin{array}{l}\text { Guzmán et al., } \\
2013^{21} \\
\qquad \mathrm{Ir} \\
\text { Isi }\end{array}$ & $\begin{array}{l}\text { Registro del Departamento } \\
\text { de Medicina Materno-fetal del } \\
\text { Instituto Nacional de Perinatología } \\
\text { sidro Espinosa de los Reyes, México }\end{array}$ & 1993-2010 & 5 por $10000 \mathrm{RNV}$ & $\begin{array}{c}\text { Relación 1:1,15 } \\
\text { (masculino/ femenino). } \\
\text { La prevalencia no está } \\
\text { calculada sobre la base de } \\
\text { un registro poblacional. }\end{array}$ \\
\hline
\end{tabular}

ABS: secuencia de bandas amnióticas; RNV: recién nacidos vivos; BWD: defectos de la pared corporal; LBWC: complejo pared corporal-extremidades. 
permitía que el contenido abdominal se ubicara en la cavidad celómica extraembrionaria. ${ }^{36,37}$ Las anomalías faciales y en las extremidades las atribuyeron a un defecto en las placodas craneofaciales, mientras que las anomalías internas las explicaron por la alteración de las placodas abdominales laterales que causaban deficiencia del mesodermo intermedio. ${ }^{33,37}$ Las objeciones a esta teoría son que el mesodermo y el endodermo derivan de la migración interna de los epiblastos a lo largo de la estría primitiva y no son inducidos por el ectodermo, ${ }^{33,38}$ las extremidades se originan del mesodermo con la formación secundaria de pliegues ectodérmicos apicales y los órganos internos derivan de la migración interna de epiblastos en la estría primitiva (progenitores del mesodermo). ${ }^{33,39}$ Hartwig y cols., ${ }^{37}$ también sugirieron la probabilidad de recurrencia familiar, situación que fue considerada en otros reportes de casos. ${ }^{6,19,28,40-44}$ Kruszka y cols., ${ }^{13}$ incluso, reportaron sobre un paciente con características clínicas de LBWC, en quien se identificó una mutación de novo heterocigota no sinónima en el gen IQCK (del inglés IQ Motif containing K) (c.667C>G;p.Q223E).

\section{Teoría de la disrupción amniótica}

Torpin ${ }^{7}$ propuso la ruptura o desgarre amniótico con la pérdida temporal de líquido amniótico (reabsorción inicial por el corion) y la salida de una parte o todo el feto hacia la cavidad celómica extraembrionaria con la formación de bandas fibrosas mesodérmicas entre la cara externa del corion y el amnios, que, al contactar con el feto, causarían las alteraciones en el desarrollo (constricciones y/o amputaciones, así como hendiduras faciales secundarias). ${ }^{7,35}$ Higginbottom y colaboradores ${ }^{1}$ apoyaron esta teoría y propusieron que un daño temprano (antes de los 45 días de gestación) producía hendiduras craneofaciales y anomalías graves del cráneo y del cerebro, con anillos de constricción y amputaciones frecuentes, pero no siempre presentes, mientras que, en el daño tardío (después de los 45 días de gestación), había mayor involucramiento de las extremidades. Sugirieron que los defectos del tubo neural y las hendiduras faciales se debían a la disrupción por bandas y / o compresión, mientras que los BWD fueron considerados una malformación primaria. ${ }^{1,35,45,46}$ Davies y cols. ${ }^{47}$ reportaron una asociación de adhesiones amnióticas al feto y múltiples malformaciones, las cuales ocurrían, predominantemente, en la misma área y del mismo lado, lo que apoyaba la teoría de las adhesiones amnióticas como el factor inicial.

Romero y colaboradores ${ }^{48}$ sostuvieron la generación de ABS por la ruptura temprana de las membranas amnióticas y coriónicas, además de proponer la transición epitelio-mesénquima como un factor que podía contribuir a la patogénesis de la ABS. ${ }^{49-51}$ Las objeciones a la teoría de la disrupción amniótica son que no hay evidencia de la reabsorción inicial del líquido amniótico por el corion o de la naturaleza abrasiva del corion sobre la piel fetal y la presencia de anomalías sin evidencia de ruptura amniótica o bandas amnióticas, el tiempo de la gestación en que ocurre la lesión debería ser muy temprano para explicar algunas de las anomalías asociadas y la presencia de malformaciones internas (cardíacas y renales) no pueden explicarse por el fenómeno mecánico. 33,35,52,53,54

\section{Teoría de la disrupción vascular}

Van Allen y colaboradores ${ }^{18}$ propusieron que los eventos internos o externos causaban accidentes vasculares o influían negativamente en el aporte sanguíneo embrionario, ya que interrumpían la morfogénesis o destruían estructuras, mientras que las bandas amnióticas constituían una forma de necrosis superficial. Esta teoría fue apoyada por algunos experimentos en modelos animales que produjeron anomalías después de una punción amniótica ${ }^{55,56}$ y por la demostración de alteraciones vasculares proximales a la banda o a la amputación. ${ }^{57}$ Las objeciones a esta teoría son que, en humanos, no ocurre la pérdida inmediata de líquido amniótico después de la ruptura de la membrana amniótica durante el embarazo temprano, de tal forma que no se esperan cambios vasculares que expliquen todas las alteraciones observadas en la ABS. ${ }^{37}$ Otra objeción es que los gemelos acárdicos (considerados como el ejemplo más claro de disrupción vascular), en comparación con los casos de ABS, muestran un patrón de anomalías diferentes, por lo que es baja la probabilidad de un mismo mecanismo causal. ${ }^{58}$

\section{Teoría de la desorganización}

El ratón mutante para el gen de desorganización (Ds) fue descrito, inicialmente, por Hummel; ${ }^{59}$ sin embargo, dicho gen no ha sido identificado en humanos. ${ }^{60}$ Se ha propuesto que las anomalías de las extremidades en los humanos pueden ser equivalentes a las reportadas en $\operatorname{Ds}^{61-69}$ aunque algunas de las características 
observadas en la ABS no forman parte del fenotipo descrito. ${ }^{33,70}$

\section{Hipótesis de la falla ectodérmica primaria en el disco embrionario temprano}

Hunter y colaboradores ${ }^{33}$ propusieron que las anomalías observadas en el LBWC se debían a un defecto primario/deficiencia del ectodermo del disco embrionario. El área afectada y la gravedad dependerían de la localización y del tamaño del defecto en el ectodermo.

En general, ninguna de las teorías en forma individual puede explicar todas y cada una de las anomalías observadas en la ABS, por lo que se ha propuesto como una entidad multifactorial en la que pueden participar diversos procesos. ${ }^{35}$

\section{Factores de riesgo (véase la Tabla 3)}

El incremento en el riesgo de ABS en progenitores jóvenes se ha atribuido a la interacción de factores genéticos con factores ambientales relacionados con la edad (mayor exposición a tabaco, alcohol y drogas). ${ }^{72} \mathrm{El}$ tabaco tiene un efecto vasoconstrictor ${ }^{94,95}$ y el monóxido de carbono se asocia con hipoxia fetal, la misma que causa disrupción vascular. ${ }^{96,97}$ La cocaína es un potente vasoconstrictor y puede dañar el flujo uteroplacentario durante períodos críticos en el desarrollo. ${ }^{28,76}$

Cignini y cols., ${ }^{9}$ sugieren que, aun cuando el paracetamol se ha asociado a un leve incremento en el riesgo de gastrosquisis, ${ }^{9,94,98}$ el riesgo observado para ABS debe contemplar el efecto confusor que tiene el uso del fármaco en caso de fiebre, ya que esta se ha asociado con disrupción vascular, defectos del tubo neural y hendiduras orales. ${ }^{99,100}$

Respecto al incremento en la frecuencia de ABS en poblaciones que viven a mayor altitud, se ha propuesto un mecanismo de hipoxia, aunque no pueden descartarse variantes genéticas propias de dichas poblaciones. ${ }^{9}$

\section{Descripción clínica (véase la Tabla 4) \\ Anomalías en las extremidades}

Las bandas amnióticas pueden estar o no unidas a las partes anormales del feto y asociarse a anillos profundos. Algunas bandas no tienen conexión con el amnios, pero unen dos partes anormales del feto; otras bandas surgen del feto o del amnios y permanecen libres sin un punto de anclaje distal. ${ }^{102}$ Patterson ${ }^{102}$ clasificó las anomalías congénitas de las extremidades en agenesia y en anillos de constricción, mientras que Hennigan y Kuo ${ }^{115}$ clasificaron las bandas de constricción de las extremidades inferiores, dividiendo su localización en 4 zonas y graduando su profundidad. Homer y cols., ${ }^{116}$ clasificaron las bandas de constricción de las extremidades superiores basándose en la propuesta de Hennigan y Kuo. ${ }^{115}$

Defectos craneofaciales

Las hendiduras craneofaciales, habitualmente, son asimétricas y no siguen la anatomía de las hendiduras faciales; se extienden desde el labio y / o paladar hacia el cráneo y pueden estar o no conectadas a la malformación cerebral por bandas amnióticas, aunque también se han reportado casos con labio y paladar hendido "común". 47,117

TABLA 3. Factores de riesgo para la secuencia de bandas amnióticas

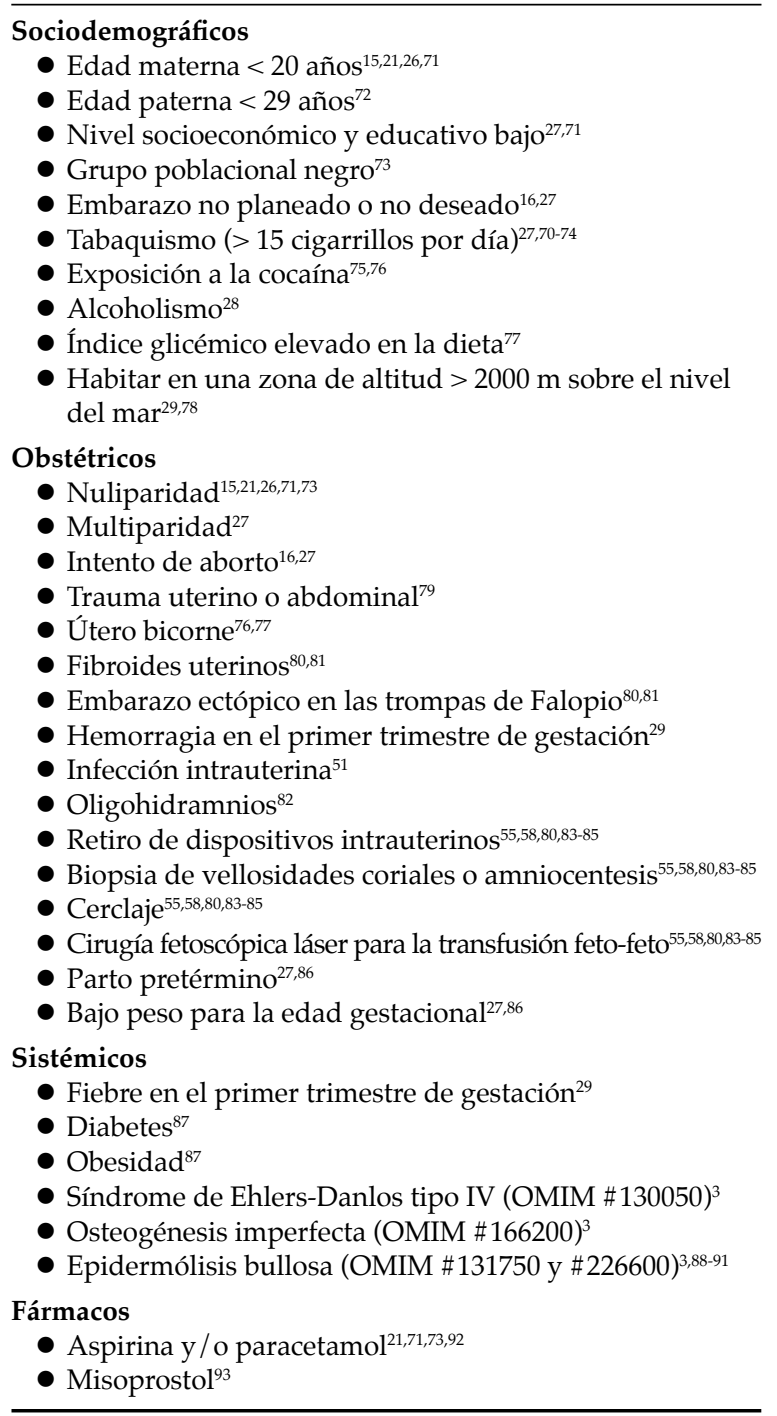

- Edad materna $<20$ años ${ }^{15,21,26,71}$

- Edad paterna $<29$ años ${ }^{72}$

- Grupo poblacional negro ${ }^{73}$

- Embarazo no planeado o no deseado ${ }^{16,27}$

- Tabaquismo (> 15 cigarrillos por día $)^{27,70-74}$

- Exposición a la cocaína ${ }^{75,76}$

Alcoholismo

Índice glicémico elevado en la dieta ${ }^{77}$

en una zona de altitud > $2000 \mathrm{~m}$ sobre el nivel stétricos

- Nuliparidad ${ }^{15,21,26,71,73}$

- Multiparidad ${ }^{27}$

- Intento de aborto ${ }^{16,27}$

Trauma uterino o abdominal

Útero bicorne ${ }^{76,7}$

- Fibroides uterinos ${ }^{80,8}$

- Embarazo ectópico en las trompas de Falopio ${ }^{80,81}$

- Hemorragia en el primer trimestre de gestación ${ }^{29}$

Infección intrauterina ${ }^{5}$

- Oligohidramnios ${ }^{82}$

Retiro de dispositivos intrauterinos ${ }^{55,58,80,83-85}$

- Biopsia de vellosidades coriales o amniocentesis $s^{55,58,80,83-85}$

- Cerclaje $\mathrm{e}^{55,58,80,83-8}$

- Cirugía fetoscópica láser para la transfusión feto-feto ${ }^{55,58,80,83-85}$

- Parto pretérmino ${ }^{27,86}$

- Bajo peso para la edad gestacional ${ }^{27,8}$

Sistémicos

Fiebre en el primer trimestre de gestación ${ }^{29}$

Diabetes $^{87}$

- Síndrome de Ehlers-Danlos tipo IV (OMIM \#130050) 3

- Osteogénesis imperfecta (OMIM \#166200)3

- Epidermólisis bullosa (OMIM \#131750 y \#226600) 3,88-9

- Aspirina y/o paracetamol $21,71,73,92$

- Misoprostol ${ }^{93}$ 
Tessier ${ }^{118,119}$ clasificó las hendiduras faciales centrándose en la órbita, con asignación de un número a cada hendidura en sentido contrario a las manecillas del reloj. David y cols., ${ }^{120}$ incrementaron la descripción de las hendiduras craneofaciales descritas por Tessier ${ }^{118}$ con el apoyo de estudios reconstructivos en segunda y tercera dimensión mediante tomografía computarizada de alta resolución.

\section{Defectos de la pared corporal y otras anomalías}

Los defectos de la pared abdominal anterior (que excluyen gastrosquisis, onfalocele y hernia umbilical) son lesiones poco comunes, grandes y complejas, ${ }^{12}$ aunque también hay bandas que no afectan la pared corporal. ${ }^{121-125}$

\section{DIAGNÓSTICO}

El análisis histológico puede mostrar la ausencia de la membrana amniótica sobre la superficie fetal del saco coriónico (que incluye la placenta), remanente de amnios en la base del cordón umbilical y detritus celulares o lamelas amnióticas embebidas en la superficie del corion. ${ }^{126,127}$
Los estudios de imagen permiten la localización, el tipo y la amplitud de las anomalías. La resonancia magnética evalúa la profundidad de las constricciones, la extensión del linfedema, la integridad muscular y define la anatomía vascular (la cual puede ser anómala) para proponer el abordaje quirúrgico y prevenir el daño vascular durante la cirugía. ${ }^{127,128}$

Las herramientas citogenéticas y moleculares, además de descartar alteraciones cromosómicas numéricas o estructurales (estudio citogenético convencional con bandas $G$, hibridación por fluorescencia in situ [Fluorescence In Situ Hybridization; FISH, por sus siglas en inglés]) y desbalances genómicos (hibridación genómica comparativa [Comparative Genomic Hybridization; CGH, por sus siglas en inglés]), y el análisis del número de copias mediante microarreglos cromosómicos de polimorfismos de nucleótido sencillo (Chromosomal Single Nucleotide Polymorphism Microarray) ${ }^{129}$ permiten la identificación de variantes comunes, raras o nuevas en el ácido desoxirribonucleico (ADN) con riesgo elevado de ABS. Tal es el caso de la secuenciación del exoma; sin embargo, esta ha

Tabla 4. Anomalías identificadas en la secuencia de bandas amnióticas

\begin{tabular}{|c|c|c|}
\hline \multirow[t]{4}{*}{ Extremidades $^{6,8,22,33,101-105}$} & Deformación & Dislocación de cadera, pie equino varo, pie talo valgo \\
\hline & Deficiencias & Completas \\
\hline & & Parciales (terminal, transversal e intercalar) \\
\hline & Digitales & $\begin{array}{l}\text { Sindactilia, seudosindactilia, camptodactilia, polidactilia, braquidactilia, } \\
\text { hipoplasia ungueal, dermatoglifos alterados }\end{array}$ \\
\hline \multirow[t]{6}{*}{ Cabeza y cuello ${ }^{9,22,28,29,33,106-114}$} & $\begin{array}{l}\text { Cabeza y } \\
\text { sistema nervioso } \\
\text { central }\end{array}$ & $\begin{array}{l}\text { Encefalocele, exencefalia, acefalia, anencefalia/acrania, } \\
\text { holoprosencefalia, microcefalia, ventriculomegalia y/o hidrocefalia, } \\
\text { displasia septo/óptica, heterotopia nodular periventricular, } \\
\text { polimicrogiria, displasia de la corteza cerebral con giros amplios, } \\
\text { hipoplasia de cuerpo calloso, craneosinostosis, mielocele, meningocele, } \\
\text { mielomeningocele }\end{array}$ \\
\hline & Cara & Asimetría, agnatia, hendiduras \\
\hline & Ojos & $\begin{array}{l}\text { Hipertelorismo orbitario, coloboma palpebral, anoftalmía/microftalmía, } \\
\text { ectropión }\end{array}$ \\
\hline & Nariz & Atresia de coanas \\
\hline & Boca & $\begin{array}{l}\text { Microstomía, paladar alto arqueado, aglosia/microglosia, labio y/o } \\
\text { paladar hendido }\end{array}$ \\
\hline & Orejas & Implantación baja o rotación, pinna mal diferenciada, melotia \\
\hline \multirow[t]{7}{*}{ Otras $^{9,10,16,22,28,29,106}$} & Cardiovascular & Anomalías cardíacas \\
\hline & Respiratorio & Anomalías pulmonares \\
\hline & Piel & $\begin{array}{l}\text { Apéndices dérmicos, pedículos hamartomatosos, pterigium, } \\
\text { apéndice sacrococcígeo }\end{array}$ \\
\hline & Tórax & Toracosquisis, pezones supernumerarios \\
\hline & $\begin{array}{l}\text { Abdominal y } \\
\text { gastrointestinal }\end{array}$ & $\begin{array}{l}\text { Abdominosquisis, fístula traqueoesófagica, defecto diafragmático, } \\
\text { hepatomegalia, malrotación intestinal, arteria umbilical única, } \\
\text { cordón umbilical corto o ectópico, ano imperforado }\end{array}$ \\
\hline & Genitourinario & Epispadias, extrofia vesical, extrofia de cloaca \\
\hline & Esquelético & $\begin{array}{l}\text { Costillas cervicales, defectos de segmentación vertebral, } \\
\text { cifoescoliosis, artrogriposis }\end{array}$ \\
\hline
\end{tabular}


sido exitosa en la identificación de mutaciones en fenotipos predominantemente mendelianos con alelos muy penetrantes, mientras que la ABS puede ser causada por una combinación de variantes en el $A D N$, por lo que se requiere el análisis familiar y la evaluación de las vías funcionales que pueden estar involucradas en el desarrollo de ABS para seleccionar las variantes que deben ser validadas mediante secuenciación de Sanger y, entonces, replicar la secuenciación en la familia o la población control. ${ }^{130} \mathrm{El}$ uso de estas herramientas puede permitir el incremento del conocimiento sobre las bases genéticas y ambientales que participan en el desarrollo de esta entidad. ${ }^{130}$

\section{DIAGNÓSTICO PRENATAL}

El ultrasonido de segunda dimensión permite la detección del LBWC en el segundo trimestre de la gestación; sin embargo, a diferencia del ultrasonido de tercera dimensión, tiene algunas limitaciones para desplegar imágenes ortogonales multiplanares, de la cavidad y de la superficie, que permiten visualizar el defecto con las estructuras adyacentes. ${ }^{131-134}$ Un marcador prenatal de ultrasonido sugestivo de ABS es el amnios libre en la cavidad, ${ }^{135-137}$ por lo que debe realizarse una evaluación estructural fetal para descartar otras anomalías. ${ }^{18,19,33,109,131,134,138}$

Cuando se identifica una imagen ecográfica sugestiva de banda amniótica, debe hacerse el diagnóstico diferencial con lo siguiente:

a) Lóbulo succenturiado de la placenta: masa separada de vellosidades coriónicas conectadas con el resto de la placenta a través de vasos dentro de las membranas. $\mathrm{Al}$ aplicar el ultrasonido doppler, presenta flujo sanguíneo. ${ }^{139}$ b) Adherencias intrauterinas: se observa una doble capa de amnios y corion. Al aplicar el ultrasonido doppler, hay vascularidad con pulso arterial concordante con la frecuencia cardíaca materna. ${ }^{140-142}$

- Sinequia: proceso cicatricial intrauterino secundario a legrados o cirugías.

- Sábana amniótica: sinequia que no tiene relación con las paredes laterales uterinas y está rodeada completamente por el saco amniótico y corion (en la forma incompleta, hay un borde libre flotante ovalado o signo del "espermio"). ${ }^{140,143}$

c) Tabique uterino: remanente del tabique medio secundario a la alteración mulleriana. ${ }^{139}$ Está localizado en el fondo uterino, con base más ancha que el extremo libre, no tiene contacto con el feto y, en su interior, es posible identificar flujo vascular de origen materno. ${ }^{140}$ Cuando las bandas amnióticas se asocian con anomalías fetales, debe descartarse teratogénesis (que incluye infeccioneos intrauterinas por Toxoplasma, virus de la rubéola, citomegalovirus, herpes y otras.) y alteraciones cromosómicas. ${ }^{131}$ También deben considerarse, en el diagnóstico diferencial, entidades monogénicas, como el síndrome de Adams-Oliver (OMIM \#100300, gen ARHGAP31; OMIM \#616028, gen NOTCH1; OMIM \#614219, gen DOCK6) ${ }^{3}$ y el síndrome de Roberts (OMIM \#268300). ${ }^{3}$

\section{Manejo}

Requiere un abordaje multidisciplinario. Debe realizarse vigilancia con ultrasonido para determinar si las bandas amnióticas (en caso de estar presentes) presentan lisis espontánea, ${ }^{144,145}$ si el proceso está causando deformación fetal o si es necesario liberar intrauterinamente las bandas de constricción mediante fetoscopia. ${ }^{146-150}$

TABLA 5. Clasificación prenatal por estadios de la secuencia de bandas amnióticas que involucra las extremidades ${ }^{143,144}$

\begin{tabular}{ll}
\hline Estadio & Descripción \\
\hline 1 & Bandas amnióticas sin signos de constricción. \\
2 & Constricción sin compromiso vascular (ultrasonido doppler normal cuando se compara con la extremidad \\
contralateral); puede tener deformidad distal: & A. Sin o leve linfedema. \\
& B. Con linfedema grave. \\
3 & Constricción grave con compromiso arterial progresivo. El flujo debe medirse proximal y distalmente al sitio de la \\
& banda de constricción. \\
& A. Ultrasonido doppler distal anormal cuando se compara con la extremidad contralateral. \\
4 & Curvatura o fractura de huesos largos en el sitio de constricción. \\
5 & Amputación intrauterina.
\end{tabular}

En los estadios 2-5, las bandas amnióticas pueden ser o no visualizadas mediante ultrasonido. 
Hüsler y cols., ${ }^{150}$ tomando como referencia la clasificación posnatal sugerida por Weinzweig, ${ }^{151}$ propusieron la clasificación prenatal de los estadios de la ABS que afectaban las extremidades mediante la evaluación de la velocimetría doppler e índice de pulsatilidad arterial (Tabla 5). ${ }^{150}$ Los fetos que pueden beneficiarse de la liberación de bandas amnióticas mediante fetoscopia son los que tienen flujo anormal (al compararlo con la extremidad contralateral o con valores de referencia disponibles) en la porción distal de la extremidad. ${ }^{150,152,153} \mathrm{El}$ objetivo del procedimiento es evitar lesiones irreversibles y tener la oportunidad de contar con una extremidad reconstruible y funcional en la vida posnatal. ${ }^{153}$

Cuando el diagnóstico se efectúa posnatalmente, la clasificación propuesta por Hennigan y $\mathrm{Kuo}^{115}$ es utilizada para la elección de la cirugía, es decir, la reparación de tipo estético y electivo en casos con bandas superficiales y sin compromiso del drenaje circulatorio o linfático, y el tratamiento emergente en los casos con bandas profundas que comprometen la integridad anatómica y funcional del sitio afectado. ${ }^{106}$ Las anomalías craneofaciales y de la pared corporal deben ser abordadas quirúrgicamente de acuerdo con las guías de práctica clínica según cada caso. ${ }^{154}$

\section{Pronóstico}

Depende del momento del diagnóstico (la mayoría se efectúa posnatalmente), del tipo y de la localización de las anomalías, y puede variar desde repercusiones cosméticas hasta incompatibilidad con la vida. ${ }^{21} \mathrm{El}$ diagnóstico prenatal de adherencias amnióticas se ha asociado con un pronóstico adverso. La mayoría de los defectos craneofaciales y de la pared corporal son incompatibles con la vida extrauterina. ${ }^{127}$ En cuanto a la presencia de bandas amnióticas, el factor pronóstico fetal más importante es la perfusión de la porción distal de la extremidad involucrada. ${ }^{150}$ Se ha reportado la muerte fetal por la estrangulación del cordón umbilical con una banda amniótica, 2,155,156 aunque también se ha reportado la resolución espontánea de las bandas de constricción. ${ }^{145,150}$

En casos de diagnóstico posnatal con anomalías limitadas a las extremidades, el pronóstico es bueno después de la reparación quirúrgica.

\section{Asesoramiento genético}

Hasta el momento, la ABS es considerada un evento esporádico, probablemente, multifactorial, con un riesgo de recurrencia similar al de la población general $(<1 \%)$, aunque se han reportado casos familiares en los que el riesgo debe ser calculado para cada familia en particular. ${ }^{6,28,40-44}$

\section{DISCUSIÓN}

La mayor prevalencia de ABS en fetos $<20$ semanas de gestación y óbitos implica un subdiagnóstico de esta entidad. Se han reportado diversos factores de riesgo y una gran variabilidad de las manifestaciones clínicas, así como en el pronóstico. Actualmente, es posible efectuar el diagnóstico prenatal de esta entidad con los potenciales beneficios que este implica, en particular, ante la presencia de bandas amnióticas que pueden ser liberadas mediante fetoscopia con la finalidad de evitar lesiones irreversibles y tener la oportunidad de efectuar la reconstrucción de la extremidad afectada.

\section{REFERENCIAS}

1. Higginbottom MC, Jones KL, Hall BH, et al. The amniotic band disruption complex: timing of amniotic rupture and variable spectra of consequent defects. I Pediatr 1979;94(4):544-9.

2. Kalousek DK, Bamforth $S$. Amnion rupture sequence in previable fetuses. Am J Med Genet 1988;31(1):63-73.

3. OMIM Online Mendelian Inheritance in Man. An online catalog of human genes and genetic disorders. [Acceso: 31 de enero de 2017]. Disponible en: https: / / www.omim.org.

4. Hennekam RC, Biesecker LG, Allanson JE, et al. Elements of morphology: general terms for congenital anomalies. Am J Med Genet A 2013;161A(11):2726-33.

5. Seeds JW, Cefalo RC, Herbert WN. Amniotic band syndrome. Am J Obstet Gynecol 1982;144(3):243-8.

6. Levy R, Lacombe D, Rougier Y, et al. Limb body wall complex and amniotic band sequence in sibs. Am J Med Genet A 2007;143A(22):2682-7.

7. Torpin R. Amniochorionic mesoblastic fibrous strings and amniotic bands: associated constricting fetal malformations or fetal death. Am J Obstet Gynecol 1965;91:65-75.

8. Bodamer OA, Popek EJ, Bacino C. Atypical presentation of amniotic band sequence. Am J Med Genet 2001;100(2):100-2.

9. Cignini P, Giorlandino C, Padula F, et al. Epidemiology and risk factors of amniotic band syndrome, or ADAM sequence. J Prenat Med 2012;6(4):59-63.

10. Jamsheer A, Materna-Kiryluk A, Badura-Stronka M, et al. Comparative study of clinical characteristics of amniotic rupture sequence with and without body wall defect: further evidence for separation. Birth Defects Res A Clin Mol Teratol 2009;85(3):211-5.

11. Martínez-Frías ML. Epidemiological characteristics of amniotic band sequence (ABS) and body wall complex (BWC): are they two different entities? Am J Med Genet 1997;73(2):176-9.

12. Martínez-Frías ML. Clinical and epidemiological characteristics of infants with body wall complex with and without limb deficiency. Am J Med Genet 1997;73(2):170-5.

13. Kruszka P, Uwineza A, Mutesa L, et al. Limb body wall complex, amniotic band sequence, or new syndrome caused by mutation in IQ Motif containing K (IQCK)? Mol Genet 
and Genomic Med 2015;3(5):424-32.

14. Herrmann J, Opitz JM. Naming and nomenclature of syndromes. Birth Defects Orig Artic Ser 1974;10(7):69-86.

15. Garza A, Cordero JF, Mulinare J. Epidemiology of the early amnion rupture spectrum of defects. Am J Dis Child 1988;142(5):541-4.

16. Lowry RB, Bedard T, Sibbald B. The prevalence of amnion rupture sequence, limb body wall defects and body wall defects in Alberta 1980-2012 with a review of risk factors and familial cases. Am J Med Genet A 2017;173(2):299-308.

17. Pagon RA, Stephens TD, McGillivray BC, et al. Body wall defects with limb reduction anomalies: a report of fifteen cases. Birth Defects Orig Artic Ser 1979;15(5A):171-85.

18. Van Allen MI, Curry C, Gallagher L. Limb body wall complex: I Pathogenesis. Am J Med Genet 1987;28(3):529-48.

19. Moerman P, Fryns JP, Vandenberghe K, et al. Constrictive amniotic bands, amniotic adhesions, and limb-body wall complex: discrete disruption sequences with pathogenic overlap. Am J Med Genet 1992;42(4):470-9.

20. Russo R, D’Armiento M, Angrisani P, et al. Limb body wall complex: a critical review and a nosological proposal. $A m$ J Med Genet 1993;47(6):893-900.

21. Guzmán-Huerta ME, Muro-Barragán SA, AcevedoGallegos S, et al. Amniotic band sequence: prenatal diagnosis, phenotype descriptions, and a proposal of a new classification based on morphologic findings. Rev Invest Clin 2013;65(4):300-6.

22. Opitz JM, Johnson DR, Gilbert-Barness EF. ADAM "sequence" part II: hypothesis and speculation. Am J Med Genet A 2015;167A(3):478-503.

23. EUROCAT European surveillance of congenital anomalies. [Acceso: 30 de enero de 2017]. Disponible en: http:/ / www. eurocat-network.eu.

24. Froster UG, Baird PA. Amniotic band sequence and limb defects: data from a population-based study. Am J Med Genet 1993;46(5):497-500.

25. Ossipoff V, Hall BD. Etiologic factors in the amniotic band syndrome: a study of 24 patients. Birth Defects Orig Artic Ser 1977;13(3D):117-32.

26. Bower C, Norwood F, Knowles S, et al. Amniotic band syndrome: a population-based study in two Australian states. Paediatr Perinat Epidemiol 1993;7(4):395-403.

27. Czeizel AE, VitézM, KodajI, etal.Study of isolated apparent amniogenic limb deficiency in Hungary, 1975-1984. Am J Med Genet 1993;46(4):372-8.

28. Luehr B, Lipsett J, Quinlivan JA. Limb-body wall complex: a case series. J Matern Fetal Neonatal Med 2002;12(2):132-7.

29. OrioliIM, RibeiroMG, CastillaEE.Clinicalandepidemiological studies of amniotic deformity, adhesion, and mutilation (ADAM) sequence in a South American (ECLAMC) population. Am J Med Genet A 2003;118A(2):135-45.

30. Koskimies E, Syvänen J, Nietosvaara Y, et al. Congenital constriction band syndrome with limb defects. J Pediatr Orthop 2015;35(1):100-3.

31. LuebkeHJ, ReiserCA,PauliRM. Fetal disruptions: assessment of frequency, heterogeneity, and embryologic mechanisms in a population referred to a community-based stillbirth assessment program. Am J Med Genet 1990;36(1):56-72.

32. Streeter GL. Focal deficiencies in fetal tissues and their relation to intra-uterine amputation. Washington: Carnegie Institution of Washington;1930.

33. Hunter AG, Seaver LH, Stevenson RE. Limb-body wall defect. Is there a defensible hypothesis and can it explain all the associated anomalies? Am J Med Genet A 2011;155A(9):2045-59.

34. McKenzie J. Amniotic bands. En: Balls M, Wild E, eds. The early development of mammals, the second symposium of the British Society for Developmental Biology. New York:
Cambridge University Press; 1975.

35. Halder A. Amniotic band syndrome and/or limb body wall complex: split or lump. Appl Clin Genet 2010;3:7-15.

36. Smits-Van Prooije AE, Vermeij-Keers C, Poelmann RE, et al. The formation of mesoderm and mesectoderm in 5-to41 somite rat embryos cultured in vitro, using WGA-Au as a marker. Anat Embryol (Berl) 1988;177(3):245-56.

37. Hartwig NG, Vermeij-Keers C, De Vries HE, et al. Limb body wall malformation complex: an embryologic etiology? Hum Pathol 1989;20(11):1071-7.

38. Wei X, Sulik KK. Pathogenesis of craniofacial and body wall malformations induced by ochrotoxin $\mathrm{A}$ in mice. Am J Med Genet 1993;47(6):862-71.

39. Sadler TW. Langman's medical embriology. 12th ed. Philadelphia:Wolters Kluwer Health/LippincottWilliams \& Wilkins; 2012 .

40. Lubinsky M, Sujansky E, Sanger W, et al. Familial amniotic bands. Am J Med Genet 1983;14(1):81-7.

41. Gellis SS. Constrictive bands in the human. Birth Defects Orig Artic Ser 1977;13(1):259-68.

42. Temtamy SA, McKusick VA. Digital and other malformations associated with congenital ring constrictions. Birth Defects Orig Artic Ser 1978;14:547.

43. Etches PC, Stewart AR, Ives EJ. Familial congenital amputations. J Pediatr 1982;101(3):448-9.

44. Lockwood C, Ghidini A, Romero R. Amniotic band syndrome in monozygotic twins: prenatal diagnosis and pathogenesis. Obstet Gynecol 1988;71(6 Pt 2):1012-6.

45. Bhat BV, Pandey KK, Srinivasan S, et al. Intrauterine amputation associated with amniotic band syndrome. Indian Pediatr 1991;28(2):188-91.

46. Jabor MA, Cronin ED. Bilateral cleft lip and palate and limb deformities: a presentation of amniotic band sequence? J Craniofac Surg 2000;11(4):388-93.

47. Davies BR, Giménez-Scherer JA, Hernández-Sierra JF. Fetal amniotic adhesions. Their topographic concordance with regionally clustered malformations. Arch Med Res 2001;32(1):48-61.

48. Romero-Valdovinos M, Galván-Montaño A, Olivo-Díaz A, et al. The amniotic band syndrome in the rat is associated with the activation of transforming growth factor-B. Am J Pathol 2015;185(8):2076-82.

49. Eckes B, Zigrino P, Kessler D, et al. Fibroblast-matrix interactions in wound healing and fibrosis. Matrix Biol 2000;19(4):325-32.

50. Yeh YC, Wei WC, Wang YK, et al. Transforming growth factor-beta 1 induces Smad3-dependent beta 1 integrin gene expression in epithelial-to-mesenchymal transition during chronic tubulointerstitial fibrosis. Am J Pathol 2010;177(4):1743-54.

51. Romero-Valdovinos M, Bobadilla-Sandoval N, Flisser A, et al. The epithelial mesenchymal transition process may contribute to the pathogenesis of amniotic band syndrome. Med Hypotheses 2014;83(3):306-11.

52. Herva R, Karkinen-Jääskeläinen M. Amniotic adhesion malformation syndrome: fetal and placental pathology. Teratology 1984;29(1):11-9.

53. Yang SS, Levine AJ, Sanborn JR, et al. Amniotic rupture, extra-amniotic pregnancy, and vernix granulomata. Am J Surg Pathol 1984;8(2):117-22.

54. Clavert JM, Clavert A, Berlizon A, et al. Abnormalities resulting from intra-adnexal injection of glucose in the rabbit embryo-an experimental model of "amniotic disease". Prog Pediatr Sur 1978;12:143-64.

55. Kino Y. Clinical and experimental studies of the congenital constriction band syndrome with emphasis on its etiology. J Bone Joint Sur Am 1975;57(5):636-43.

56. Kennedy LA, Persaud TV. Pathogenesis of developmental 
defects induced in the rat by amniotic sac puncture. Acta Anat (Basel) 1977;97(1):23-35.

57. Daya M, Makakole M. Congenital vascular anomalies in amniotic band syndrome of the limbs. J Pediatr Surg 2011;46(3):507-13.

58. Davies BR, Giménez-Scherer JA. Comparison of the amniotic band disruption complex with acardiac twins does not support its vascular origin. Fetal Pediatr Pathol 2007;26(2):87-99.

59. Hummel KP. The inheritance and expression of disorganization, an unusual mutation in the mouse. J Exp Zool 1958;137(3):389-423.

60. Robin NH, Abbadi N, McCandlessSE, et al. Disorganization in mice and humans and its relation to sporadic birth defects. Am J Med Genet 1997;73(4):425-36.

61. Donnai D, Winter RM. Disorganisation: a model for 'early amnion rupture'? J Med Genet 1989;26(7):421-5.

62. Crosby JL, Varnum DS, Nadeau JH. Two-hit model for sporadic congenital anomalies in mice with the disorganization mutation. Am J Hum Genet 1993;52(5): 866-74.

63. Isidor B, Baujat G, Le Caignec C, et al. Congenital skin pedicles with or without amniotic band sequence: extending the human phenotype resembling mouse disorganization. Am J Med Genet A 2009;149A(8):1734-9.

64. Purandare SM, Ernst L, Medne L, et al. Developmental anomalies with features of disorganization (Ds) and amniotic band sequence (ABS): a report of four cases. $A m$ J Med Genet A 2009;149A(8):1740-8.

65. Lowry RB, Yong SL. Cleft lip and palate, sensorineural deafness, and sacral lipoma in two brothers: a possible example of the disorganisation mutant. J Med Genet 1991;28(2):135-7.

66. Nakamura K, Nanjyo B. Congenital skin tube pedicle associated with the constriction band syndrome. Plastic Reconstr Surg 1992;89(4):746-50.

67. Corona-Rivera JR, Corona-Rivera E, Navarro-Ramírez P, et al. Lumbopedal skin pedicle in an infant with amniotic band syndrome: a disorganization-like defect? Clin Dysmorphol 2003;12(1):51-4.

68. Onal EE, Türkylmaz C, Atalay $\mathrm{Y}$, et al. Tubular skin appendage, renal agenesis and popliteal web: a further example of the human homologue of disorganization (Ds). Clin Dysmorphol 2005;14(2):89-91.

69. Robin NH, Franklin J, Prucka S, et al. Clefting, amniotic bands, and polydactyly: a distinct phenotype that supports an intrinsic mechanism for amniotic band sequence. $A m \mathrm{~J}$ Med Genet A 2005;137A(3):298-301.

70. Heyne TF, Robin NH, Lin AE. Sxiteenth-century German woodcut of a male infant with possible disorganization. Clin Genet 2016;89(2):269-71.

71. Werler MM, Louik C, Mitchell AA. Epidemiologic analysis of maternal factors and amniotic band defects. Birth Defects Res A Clin Mol Teratol 2003;67(1):68-72.

72. Grewal J, Carmichael SL, Yang W, et al. Paternal age and congenital malformations in offspring in California, 19892002. Matern Child Health J 2012;16(2):385-92.

73. Werler MM, Bosco JL, Shapira SK. Maternal vasoactive exposures, amniotic bands and terminal transverse limb defects. Birth Defects Res A Clin Mol Teratol 2009;85(1):52-7.

74. Hoyt AT, Canfield MA, Romitti PA, et al. Associations between maternal periconceptional exposuretosecondhand tobacco smoke and major birth defects. Am J Obstet Gynecol 2016;215(5):613.e1-11.

75. Webster WS, Brown-Woodman PD. Cocaine as a cause of congenital malformations of vascular origin: experimental evidence in the rat. Teratology 1990;41(6):689-97.

76. Viscarello RR, Ferguson DD, Nores J, et al. Limb-body wall complex associated with cocaine abuse: further evidence of cocaine's teratogenicity. Obstet Gynecol 1992;80(3Pt2):523-6.

77. Yazdy MM, Mitchell AA, Liu S, et al. Maternal dietary glycaemic intake during pregnancy and the risk of birth defects. Paediatr Perinat Epidemiol 2011;25(4):340-6.

78. Castilla EE, Lopez-Camelo JS, Campaña H. Altitude as a risk factor for congenital anomalies. Am J Med Genet 1999;86(1):9-14.

79. Webster WS, Lipson AH, Brown-Woodman PD. Uterine trauma and limb defects. Teratology 1987;35(2):253-60.

80. Graham JM, Miller ME, Stephan MJ, et al. Limb reduction anomalies and early in utero limb compression. J Pediatr 1980;96(6):1052-6.

81. Matsunaga E, Shiota K. Ectopic pregnancy and myoma uteri: teratogenic effects and maternal characteristics. Teratology 1980;21(1):61-9.

82. Vázquez Rueda F, Ayala MontoroJ, Blanco López F, et al. Síndrome de bandas de constricción congénitas. Rev Cir Infantil 1999;9(3):163-7.

83. Yang SS. ADAM sequence and innocent amniotic band: manifestations of early amnion rupture. Am J Med Genet 1990;37(4):562-8.

84. Firth HV, Boyd PA, Chamberlain P, et al. Severe limb abnormalities after chorion villus sampling at 56-66 days' gestation. Lancet 1991;337(8744):762-3.

85. Winer N, Salomon LJ, Essaoui M, et al. Pseudoamniotic band syndrome: a rare complication of monochorionic twins with fetofetal transfusion syndrome treated by laser coagulation. Am J Obstet Gynecol 2008;198(4):393.e1-5.

86. Gün I, Muhçu M, Müngen E, et al. Effect of an amniotic sheet on pregnancy outcomes. JUltrasound Med 2013;32(5): 807-13.

87. Durga R, Renukadevi TK. Amniotic band syndrome- a dreaded condition. J Clin Diagn Res 2016;10(1):QD04-5.

88. Young ID, Lindenbaum RH, Thompson EM, et al. Amniotic bands in connective tissue disorders. Arch Dis Child 1985;60(11):1061-3.

89. Marras A, Dessì C, Macciotta A. Epidermolysis bullosa and amniotic bands. Am J Med Genet 1984;19(4):815-7.

90. Katorza E, Weisz B, Sevillia J, et al. Unusual prenatal sonographic findings of epidermolysis bullosa mimicking an amniotic band constriction ring. J Ultrasound Med 2009;28(1):73-5.

91. Snadecki H, Criscione V, Jaquith A, et al. Dystrophic epidermolysis bullosa associated with amniotic band syndrome. Pediatr Dermatol 2014;31(2):212-6.

92. Hernandez RK, Werler MM, Romitti P, et al. Nonsteroidal antiinflammatory drug use among women and the risk of birth defects. Am J Obstet Gynecol 2012;206(3):228.e1-8.

93. González CH, Marques-Días MJ, Kim CA, et al. Congenital abnormalities in Brazilian children associated with misoprostol misuse in first trimester of pregnancy. Lancet 1998;351(9116):1624-7.

94. Torfs CP, Velie EM, Oechsli FW, et al. A population-based study of gastroschisis: demographic, pregnancy, and lifestyle risk factors. Teratology 1994;50(1):44-53.

95. Mitchell AA, Gilboa SM, Werler MM, et al. Medication use during pregnancy, with particular focus on prescription drugs: 1976-2008. Am J Obstet Gynecol 2011;205(1):51.e1-8.

96. Webster WS, Abela D. The effect of hipoxia in development. Birth Defects Res C Embryo Today 2007;81(3):215-28.

97. Werler MM. Teratogen update: pseudoephedrine. Birth Defects Res A Clin Mol Teratol 2006;76(6):445-52.

98. Torfs CP, Katz EA, Bateson TF, et al. Maternal medications and environmental exposures as risk factors for gastroschisis. Teratology 1996;54(2):84-92.

99. Nilsen NO. Vascular abnormalities due to hyperthermia in chick embryos. Teratology 1984;30(2):237-51. 
100.Dreier JW, Andersen AM, Berg-Beckhoff G. Systematic review and meta-analyses: Fever in pregnancy and health impacts in the offspring. Pediatrics 2014;133(3):e674-88.

101.Taub PJ, Bradley JP, Setoguchi Y, et al. Typical facial clefting and constriction band anomalies: an unusual association in three unrelated patients. Am J Med Genet 2003;120A(2):256-60.

102.Patterson TJ. Congenital ring-constrictions. Br J Plast Surg 1961;14:1-31.

103.Ribeiro MG, Castilla EE, Orioli IM. Can amputated digits point to clues about etiology? Am J Med Genet A 2004;128A(1):93-4.

104.Gold NB, Westgate MN, Holmes LB. Anatomic and etiological classification of congenital limb deficiencies. Am J Med Genet A 2011;155A(6):1225-35.

105.Little KJ, Cornwall R. Congenital anomalies of the hand--principles of management. Orthop Clin North Am 2016;47(1):153-68.

106.Pagon RA, Stephan MJ. Septo-optic dysplasia with digital anomalies. J Pediatr 1984;105(6):966-8.

107.Wu YC, Yang ML, Yuan CC. Prenatal diagnosis of anophthalmos with limb-body wall complex. Prenat Diagn 2000;20(9):769-70.

108.Pilu G, Nicolaides K, Ximenes R, et al. Diagnosis of fetal abnormalities-the 18-23 weeks scan. London: ISUOG \& Fetal Medicine Foundation; 2002.

109. Yamanouchi H, Ota T, Imataka G, et al. Congenital bilateral perisylvian syndromeassociated with congenital constriction band syndrome. J Child Neurol 2002;17(6):448-50.

110.Harrison IM, Brosnahan D, Phelan E, et al. Septo-optic dysplasia with digital anomalies- a recurrent pattern syndrome. Am J Med Genet A 2004;131A(1):82-5.

111.Stevens CA, Dobyns WB. Septo-optic dysplasia and amniotic bands: further evidence for a vascular pathogenesis. Am J Med Genet A 2004;125A(1):12-6.

112.Das G, Gayen S, Bandyopadhyay S, et al. Ethmocephaly with amniotic band syndrome. Middle East Afr JOphthalmol 2012;19(4):429-31.

113.Sarnat HB, Flores-Sarnat L. Neuroembryology and brain malformations: an overview. Handb Clin Neurol 2013;111:117-28.

114.Jagtap SV, Saini N, Jagtap S, et al. Otocephaly: Agnathiamicrostomia-synotia syndrome- a rare congenital anomaly. J Clin Diagn Res 2015;9(9):ED03-4.

115.Hennigan S, Kuo KN. Resistant talipes equinovarus associated with congenital constriction band syndrome. J Pediatr Orthop 2000;20(2):240-5.

116. Homer LE, Mishra A, McArthur P. Amniotic constriction bands: a case series and proposed new classification system. Hand Surg 2015;20(1):121-6.

117.Rowlatt U. Cleft lip and palate associated with amniotic band limb amputations in a 20 week human fetus. Cleft Palate J 1979;16(2):206-9.

118.Tessier P. Anatomical classification of facial, cranio-facial and latero-facial clefts. J Maxillofac Surg 1976;4(2):69-92.

119.Winters R. Tessier clefts and hypertelorism. Facial Plast Surg Clin North Am 2016;24(4):545-58.

120.David DJ, Moore MH, Cooter RD. Tessier clefts revisited with a third dimension. Cleft Palate J 1989;26(3):163-84.

121.Craven CM, Carey JC, Ward K. Umbilical cord agenesis in limb body wall defect. Am J Med Genet 1997;71(1):97-105.

122.Ben-Chaim J, Mathews RI, Gearhart JP. La extrofia de cloaca. Su anatomía, manejo y seguimiento. Rev Cir Infantil 1998;8:67-74.

123.Woo LL, Thomas JC, Brock JW. Cloacal exstrophy: a comprehensive review of an uncommon problem. J Pediatr Urol 2010:6(2);102-11.

124.Suzuki K, Matsumaru D, MatsushitaS, et al.Epispadias and the associated embryopathies: genetic and developmental basis. Clin Genet 2017;91(2):247-53.

125.Reinfeldt Engberg G, Mantel Ä, Fossum M, et al. Maternal and fetal risk factors for bladder exstrophy: a nationwide Swedish case-control study. J Pediatr Urol 2016;12(5): 304. e1-7.

126.Day-Salvatore DL, Guzman E, Weiberger B, et al. Genetics casebook. Amniotic band disruption sequence. J Perinatol 1995;15(1):74-7.

127. Chen H. Atlas of Genetic diagnosis and counseling. Heidelberg: Springer; 2012.

128.Laor T, Jaramillo D, Hoffer FA, et al. MR imaging in congenital lower limb deformities. Pediatr Radiol 1996;26(6):381-7.

129.De Wit MC, de Coo IF, Schot R, et al. Periventricular nodular heterotopia and distal limb deficiency: a recurrent association. Am J Med Genet A 2010;152A(4):954-9.

130. Krupp DR, Soldano KL, Garrett ME, et al. Missing genetic risk in neural tube defects: can exome sequencing yield an insight? Birth Defects Res A Clin Mol Teratol 2014;100(8):642-6.

131.Liu IF, Yu CH, Chang $\mathrm{CH}$, et al. Prenatal diagnosis of limb body-wall complex in early pregnancy using threedimensional ultrasound. Prenat Diagn 2003;23(6):513-4.

132.Chmait R, Pretorius D, Jones M, et al. Prenatal evaluation of facial clefts with two-dimensional and adjunctive threedimensional ultrasonography: a prospective trial. $A m \mathrm{~J}$ Obstet Gynecol 2002;187(4):946-9.

133.Rossi AC, Prefumo F. Correlation between fetal autopsy and prenatal diagnosis by ultrasound: a systematic review. Eur J Obstet Gynecol Reprod Biol 2016;210:201-6.

134.Emanuel PG, Garcia GI, Angtuaco TL. Prenatal detection of anterior abdominal wall defects with US. Radiographics 1995;15(3):517-30.

135.López Ramón y Cajal C. Diagnóstico prenatal del síndrome de las bandas amnióticas. Prog Obstet Ginecol 2000;43:35463.

136.Zimmer EZ, Bronshtein M. Ultrasound observation of amnion dysmorphism at $14.5-16$ weeks. Prenat Diagn 1995;15(5):447-9.

137.Mahony BS, Filly RA, Callen PW, et al. The amniotic band syndrome: antenatal sonographic diagnosis and potential pitfalls. Am J Obstet Gynecol 1985;152(1):63-8.

138. Chen CP. Prenatal diagnosis of limb-body wall complex with craniofacial defects, amniotic bands, adhesions and upper limb deficiency. Prenat Diagn 2001;21(5):418-9.

139. Galeana Castillo C, Casas Patiño D, Rodríguez Torres A, et al. Síndrome de bandas amnióticas: correlación ecográfica y pieza anatómica. Arch Inv Mat Inf 2013;5(3):146-50.

140.Pons GA, Sáez AR, Sepúlveda LW. Brida amniótica, sinequia intrauterina y tabique mulleriano: Etiopatogenia, diagnóstico diferencial y pronóstico. Rev Chil Ultrason 2005;8(2):51-8.

141.Abuhamad AZ, Romero R, Shaffer WK, et al. The value of Doppler flow analysis in the prenatal diagnosis of amniotic sheets. J Ultrasund Med 1992;11(11):623-4.

142.Sherer DM, Lysikiewicz AJ. Doppler flow velocimetry assisted diagnosis of an intrauterine synechia during pregnancy. Am J Perinatol 2002;19(8):421-6.

143.Zaldivar Pascua Y. Caso interesante de lámina amniótica. Rev Med Hondur 2012;80(3):111-3.

144.Nishi T, Nakano R. Amniotic band syndrome: serial ultrasonographic observations in the first trimester. J Clin Ultrasound 1994;22(4):275-8.

145.Pedersen TK, Thomsen SG. Spontaneous resolution of amniotic bands. Ultrasound Obstet Gynecol 2001;18(6):673-4.

146.Quintero RA, Morales WJ, Phillips J, et al. In utero lysis of amniotic bands. Ultrasound Obstet Gynecol 1997;10(5): 316-20. 
147.Keswani SG, Johnson MP, Adzick NS, et al. In utero limb salvage: fetoscopic release of amniotic bands for threatened limb amputation. J Pediatr Surg 2003;38(6):848-51.

148.Sentilhes L, Verspyck E, Eurin D, et al. Favourable outcome of a tight constriction band secondary to amniotic band syndrome. Prenat Diagn 2004;24(3):198-201.

149.Ronderos-Dumit D, BriceñoF, NavarroH, etal.Endoscopic release of limb constriction rings in utero. Fetal Diagn Ther 2006;21(13):255-8.

150.Hüsler MR, Wilson RD, Horii SC, et al. When is fetoscopic release of amniotic bands indicated? Review of outcome of cases treated in utero and selection criteria for fetal surgery. Prenat Diagn 2009;2(5)9:457-63.

151.Weinzweig N. Constriction band-induced vascular compromise of the foot: classification and management of the "intermediate" stage of constriction-ring syndrome. Plast Reconstr Surg 1995;96(4):972-7.
152. Wisser J, Kurmanavicius J, Müller C, et al. Pulsatility index in the fetal anterior tibial artery during the second half of normal pregnancy. Ultrasound Obstet Gynecol 1998;11(3):199203.

153.Soldado F, Aguirre M, Peiró JL, et al. Fetoscopic release of extremity amniotic bands with risk of amputation. J Pediatr Orthop 2009;29(3):290-3.

154.Muraskas JK, McDonnellJF, Chudik RJ, etal. Amniotic band syndrome with significant orofacial clefts and disruptions and distortions of craniofacial structures. J Pediatr Surg 2003;38(4):635-8.

155. HeifetzSA. Strangulation of the umbilical cord by amniotic bands: report of 6 cases and literature review. Pediatr Pathol 1984;2(3):285-304.

156.Armand R, Brenner DS, Sun CC. Pathologic quiz case: fetal death at 32 weeks of gestation. Arch Pathol Lab Med 2003;127(7):e317-8. 